\title{
HOW CONTACT LENSES HAVE INFLUENCED RESEARCH DEVELOPMENTS IN OPTICS AND VISION SCIENCE
}

Ian Cox, PhD

Center for Visual Science, University

of Rochester, Rochester, NY

iancoxphd@gmail.com

Pete Kollbaum OD, PhD

School of Optometry, Indiana

University, Bloomington, IN

\section{kollbaum@indiana.edu}

\section{Eric Papas PhD}

School of Optometry and Vision Sciences, University of New South Wales, NSW, Australia

e.papas@unsw.edu.au

doi: 10.14434/hindsight.v52i2.33226

\begin{abstract}
Initially, contact lenses were developed as the only viable option to provide improved vision for patients suffering from conditions which distorted the corneal surface in an irregular fashion, rendering spectacle correction an unsatisfactory option. Developments in materials, fitting methods and manufacturing technologies soon made contact lenses an appealing option for all spectacle wearers, particularly with the development of toric and multifocal lens options. Although the optical challenges of contact lenses appeared to be relatively straightforward in their early years, the development of improved in-vivo diagnostic and in-vitro metrology instrumentation led to a significantly greater understanding of the nature of the optical errors (e.g. higher order wavefront aberrations) of the eye and contact lenses and the interactions between them when worn. The features of contact lenses having extremely steep curvatures and being aligned closely to the eye's visual axis in all positions of gaze provide both unique challenges and opportunities for improving the visual performance of both physiological and pathological eyes, and have guided the efforts of significant vison science research in more recent times.
\end{abstract}

\section{Key Words}

higher order aberration, contact lens optical design, improved visual performance

\section{THE EARLY YEARS}

Although the first practical contact lenses were used as early as 1888 , glass blown shells formed individually to rest on the sclera and vault across the cornea were the norm until the 1930's.' They were relatively heavy, difficult to manufacture and prone to breakage. Therefore, they remained a rare and expensive device, only for the correction of vision in eyes suffering from ocular pathological conditions, when no other option was available. It was not until the introduction of plastic/glass hybrid lenses by Feinbloom in 1936, followed by the first all-plastic scleral lenses in 1938 using polymethylmethacrylate (PMMA), that lenses with superior safety, lightness and comfort were available.2 Although then still a rare form of vision correction, due mainly to the laborintensive methods of scleral fitting and manufacturing, the use of contact lenses increased substantially after the 1948 invention of the corneal lens by Tuohy. ${ }^{3}$ Accidentally cutting through the scleral shell at the edge of the optic zone that he was machining, Tuohy was dismayed by his time lost, and quickly reasoned that the small diameter lens created by the optic zone alone might still be viable. He tried the small diameter lens on his own eye and realized that a lens fit within the cornea could be more comfortable and provide longer wearing times than a scleral shell.

This invention of a relatively easy to fit, comfortable contact lens that could be repeatedly made with distortion-free optics became the beginning of the modern era of contact lenses and by the 1960's, corneal lenses made using PMMA could be manufactured to correct myopia, hyperopia, astigmatism, and even novel bifocal designs for presbyopia correction. Nevertheless, wearing times were often limited by the loss of vision caused by light scatter in the corneal epithelium, reported as "Sattler's Veil."Therefore, ironically, the good optics of these lenses were compromised by the poor physiological response of the cornea to typical periods of daily wear. The realization by Smelser and Ozanics ${ }^{4}$ that oxygen for corneal metabolism came directly from the atmosphere and not from the aqueous or limbal vasculature led to the beginnings of decades of research into corneal transparency ${ }^{5,6}$ and the oxygen demands of the physiological cornea under open and closed eye conditions, both with and without contact lenses. ${ }^{7-10}$ More immediate however, was a major shift in the fitting philosophy of corneal contact lenses. In particular, clinicians realized that the lens fit could be adjusted to replenish the oxygenated tear film with every blink, thus extending comfortable wearing times from just a few hours. Fitting strategies based around small lens diameters and shorter sagittal depths were then quickly adopted to maximize post-lens tear exchange with blinking. While this strategy made all day wear a reality for many patients, there was still a high number ceasing contact lens wear due to discomfort or corneal distortion leading to poor vision. It was the physiological response of the cornea to contact lens wear that became the primary focus of contact lens research during this time. With the manufacturing methods and metrology available in the 1960's, improvement in optical quality was not a consideration. 


\section{FROM HARD TO SOFT}

In 1960, Wichterle and Lim, two Czechoslovakian chemists, researched hydrogels and began to formulate the world's first "soft" contact lenses from their newly invented HEMA hydrogel material. ${ }^{11,12}$ This $38 \%$ water content material was highly flexible, more oxygen permeable and significantly more comfortable than the rigid PMMA corneal contact lenses that were available. Although working behind the "Iron Curtain", Wichterle granted exclusive Western Hemisphere rights to the new hydrogel materials and to a novel molding process, called "spin-casting," for the fabrication of hydrogel contact lenses to the National Patent Development Corp (NPD), a U.S. firm. NPD, in turn, licensed Bausch \& Lomb, Inc. to use these product and process patents. In 1971, after considerable improvement and several years of careful testing, Bausch \& Lomb obtained approval from the U.S. Food and Drug Administration to sell hydrogel lenses in the United States. ${ }^{13}$

The first lens made commercially available was the Bausch \& Lomb C-series contact lens. This lens fit the eye with considerable decentration and movement due to its 12.5 $\mathrm{mm}$ diameter modeled from the traditional hard lens fitting philosophy. Although the optics were compromised by the wildly aspheric posterior lens surface produced by the "spin-casting" manufacturing process, ${ }^{14}$ the lens was extremely comfortable compared to the PMMA corneal lenses it competed against in the marketplace. The rapid commercial acceptance of soft contact lenses quickly led to development of additional designs by Bausch \& Lomb and other companies. It was over this time that soft lenses become manufactured with larger diameters and greater sagittal depths to provide a more centered, less mobile lens fitting. In addition, spherical optical surfaces were now generated to minimize unwanted optical aberrations of those first, uncontrolled, aspheric designs. Comfort was no longer an issue for all waking hours of wear, and compared to PMMA lenses, the physiological response of the cornea was greatly improved.

Soft lenses were initially only available in spherical powers to correct myopia and later hyperopia, but by the mid to late 1970's soft lenses to correct astigmatism became available. ${ }^{15}$ Unlike rigid lenses which "mask" the astigmatic component of the cornea by replacing the toric corneal surface with a spherical one and filling the post-lens space with tear film, soft lenses largely conform to the underlying corneal shape, and so the toric shape of the cornea is transferred to the front surface of the soft lens. The addition of an astigmatic correction to the soft lens required a method of stabilization and orientation to be built into the physical shape of the lens. The most successful designs used an increasing thickness profile in the vertical meridian of the lens, allowing the squeeze force of the upper eyelid to stabilize the lens on the eye between blinks. Optical surfaces of early toric soft contact lenses were generated by squeezing the circular material "button" to an oval shape and then lathing a spherical surface. ${ }^{16}$ Releasing the button from the squeezing force then allowed the lens button to return to a circular shape, creating a toric optical surface with an oval optical zone. While successful, lenses manufactured in this way were difficult to make repeatedly, and so toric soft lenses were not widely accepted through to the mid 1980's. At this time, Bausch + Lomb developed a tool post for contact lens lathes that oscillated so the diamond tool could move in and out of the button while cutting the toric curve of their Optima Toric lenses, a technology so successful that they eventually licensed their patent to lathe manufacturers. ${ }^{17}$ Toric surfaces could now be generated without distorting the button, eliminating the inherent stress that plagued the optical quality of earlier designs and contributed to the consistency that clinicians expect with modern toric lenses. The toric generating lathes were the forerunners of today's computer numeric controlled (CNC) lathing technology that is the backbone of the contact lens industry. Ultimately many soft lenses were cast molded between two rigid plastic mold surfaces in the 1980's. This process was also adopted for toric soft lenses within a decade, to provide lenses with little lens to lens variance and high optical quality surfaces. However, the tooling creating the molds for these lenses is still generated using the same CNC lathes often used for directly lathing precise, and sometimes quite complex, lens surfaces.

\section{MULTIFOCAL LENSES FOR PRESBYOPIA}

During the 1980's and 90's the majority of research and development in both rigid and soft contact lenses focused on improving the corneal physiology and ocular compatibility of lenses and improving the comfort over a longer wearing time, even allowing patients to wear the lenses overnight, for several days at a time. Nevertheless, the correction of presbyopia was a new optical frontier for soft contact lenses during this time. Early attempts with rigid contact lenses focused on translating bifocal designs..$^{18}$ Here the lower edge of the lens interacts with the lower eyelid in downgaze so that the lens is held in position while the pupil of the eye moves from the upper portion of the optic zone (where the distance correction is located) to the lower section where the reading addition is positioned. These lens designs were quite successful due to the mobility and diameter of rigid lenses but were difficult and time consuming to fit. With the vast majority of the market wearing soft contact lenses which were relatively immobile on the eye, an easy to fit solution for presbyopia was needed.

Multifocal soft lenses designed to correct presbyopia were introduced by Bausch + Lomb and CIBA VISION ${ }^{\circ}$ in 1982. ${ }^{19}$ In B+L's case, experience with significant spherical aberration in their first lenses for myopia helped them manufacture a lens with spherical aberration aimed to expand the depth of field of the wearer. Ironically, after spending years trying to eliminate spherical aberration inherent in their spin-cast lens products they purposely reintroduced positive spherical aberration into the design of their PA1 single (center distance) add bifocal to extend the depth of focus of the wearer. The CIBA "BiSoft" bifocal lens was a center distance annular bifocal design with multiple add powers available. Both lenses were designed to provide improved distance and near vision for presbyopic patients using a "simultaneous imaging" concept. In this approach, light from both distant and near objects passes through the pupil simultaneously. Those light rays most conjugate to the retina and the field object will provide the most in-focus image on the retina, while those that are out of focus for that object-plane will reduce the contrast, and potentially even the resolution of the retinal image. In these simultaneous image lenses theoretically no movement or decentration of the lens is required to provide the visual transition from distance to near focus. The metrology technology of the 1980's (vertexometers) was able to measure the base power and the add 
for these early lenses but gave no estimate of the image quality at each of the focal points. Patients demonstrated acceptable distance and near visual acuity in the exam room, but reports of glare, haloes and poor vision in dim light, particularly while driving, led to low levels of acceptance with these and later multifocal contact lenses of the era.

An alternative form of correction for presbyopes, monovision, where single vision lenses are used to correct one eye for distance and the other for near, had been reported in the literature since the 1960's. ${ }^{20}$ Monovision is uniquely suited to contact lenses where, unlike spectacles, the magnification of the retinal image is low enough that the patient can fuse images in the two eyes without any apparent double vision. Initially, however, it was rarely prescribed amid concerns from practitioners that the compromise in binocularity was too severe and that the patients were effectively monocular at both distance and near focus. With the growing pressure for a contact lens based solution for presbyopia being driven by the advancing age of the "baby boomer" generation, the 1980's and 90's brought a large amount of research focused on the feasibility of monovision as an alternative to bifocal/multifocal contact lenses, particularly with regard to binocularity ${ }^{21}$ and visual performance ${ }^{22}$ under low contrast and/or low illumination conditions. Clinically, monovision became an accepted form of presbyopic correction with contact lenses, most commonly for add powers up to and including +1.50 $D$, after which the binocular contrast sensitivity was no better than for monocular correction. ${ }^{23}$ Novel studies designed to test the strengths and limitations of monovision in contemporary everyday tasks showed that it was a useful option where visual tasks did not require fine stereopsis. ${ }^{24,25}$

In the late 1980's and early 1990's the introduction of an alternative simultaneous image design, the diffractive lens, led to a reignited interest in bifocal contact lenses. Specifically, the unique diffractive designs of the rigid Pilkington Diffrax ${ }^{26}$ and soft Hydron Echelon ${ }^{27}$ lenses respectively, eliminated the impact of pupil size on the proportion of light dedicated to the distance and near retinal images that plagued more traditional refractive optics solutions. While clinical use demonstrated that the performance of the lenses were not pupil dependent, diffractive optics suffered from other problems. Most significant, and perhaps the main reason that they were not more successful, is that an inherent limitation of diffractive optics occurs when light is divided between the distance and near foci. At this point, a significant proportion of light (up to 20\%) is lost to other, higher orders of diffraction. ${ }^{28}$ From a practical standpoint, this light might be considered "scattered" across the retinal image, causing a reduction in contrast. ${ }^{29}$ Wearers perceived this scattering as a "greying" or a "washing out" of the visual scene and sometimes complained of reduced vision in conditions of low illumination or contrast. When combined with their relatively expensive cost at the time, these difficulties limited uptake of the concept in the marketplace. Interestingly, diffractive intraocular lenses are now quite common despite their lack of success within contact lenses.

It has been known for centuries that stenopaic viewing (i.e. through a pinhole) extends the depth of focus of the eye, behavior which makes it interesting as a potential means of correcting presbyopia. Although optically simple, stenopaic contact lenses have not found general acceptance, probably due to problems associated with poor cosmesis, as well as the significant reduction in retinal illumination that accompanies their use. ${ }^{30}$ Nevertheless, other efforts to utilize the principle of extend depth of focus have been made. These include, among others, creating a "virtual pinhole" by inducing rapidly increasing plus power radially from the central zone ${ }^{31}$ and manipulating higher order aberrations so that reasonable image quality is obtained over a range of object vergences. ${ }^{32}$ Lenses based on both of these unique approaches are currently available commerially.

\section{HIGHER ORDER WAVEFRONT ABERRATIONS \& THE PROMISE OF BETTER VISION}

The development of clinically applicable Hartmann-Schack wavefront sensors in the late 1990's provided the breakthrough needed to understand the link between the theoretical design and visual performance of single vision and multifocal soft contact lenses and the clinical reality of the retinal image quality that these lenses were providing. Early population measurements of the wavefront error of the eye of large contact lens wearing age populations, identified the presence of significant levels of higher order wavefront aberrations over and above the defocus and astigmatism that traditional refraction methods had identified. ${ }^{33}$ For many patients who exhibited large amounts of these higher order aberrations, retinal image quality was significantly compromised even with a best sphero-cylindrical correction in place, and especially under low illumination, large pupil conditions. This aberrometry technique was quickly adopted to measure the true optical performance of rigid and soft, single vision and multifocal contact lenses in-vivo, $34,35,36$ but also proved to be an ideal method to evaluate the optical performance of contact lenses off the eye. ${ }^{37}$ Wavefront sensor technology, combined with the next generation CNC lathing technology allowed the potential for new and varied optical designs for contact lenses to be imagined and developed.

Clinical and laboratory wavefront sensors became available commercially in the early 2000's and ignited significant research interest in the potential of altering the optics of contact lenses to improve vision in patients. This work has largely focused on either obtaining some improvement for the average eye within the population or more significant improvement in individual eyes with more significant higher order aberrations (e.g. older eyes and those with corneal shape-related pathologies such as keratoconus). 
$\mathrm{AO}$

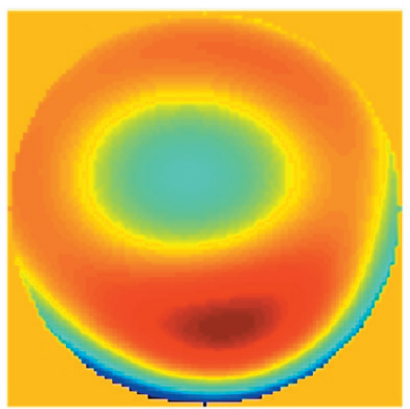

PV

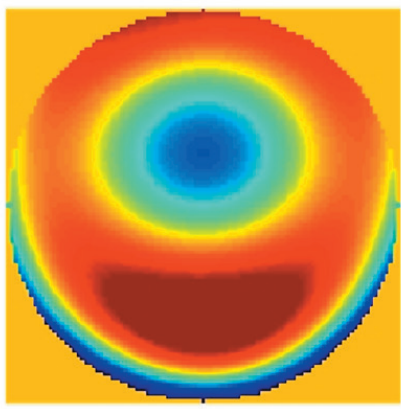

Microns

$-0.80$

0
FD

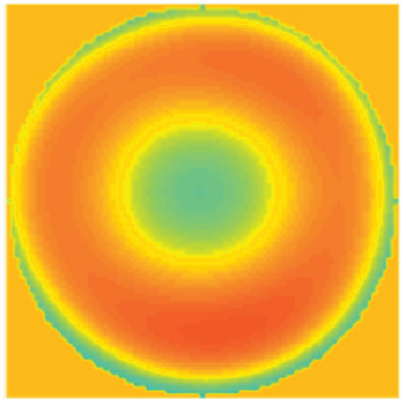

AV
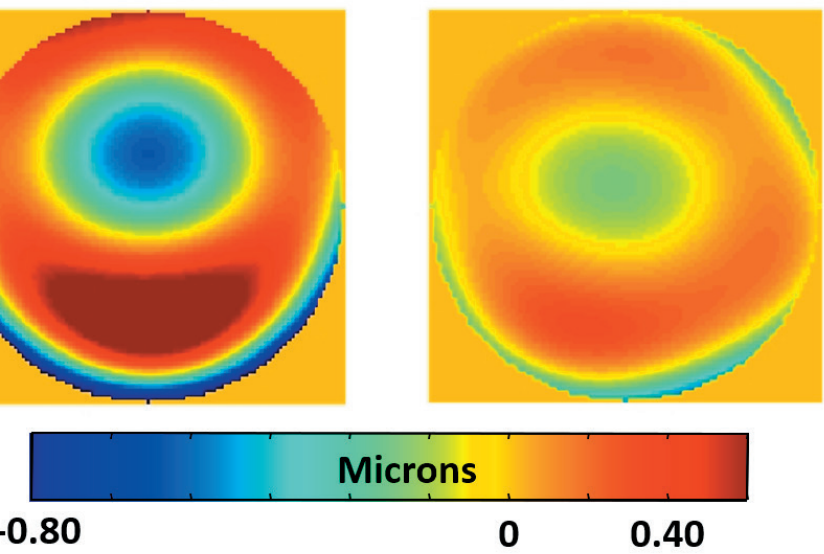

Figure 1. Higher order wavefront error maps ( $6 \mathrm{~mm}$ pupil) of 4 different soft toric contact lenses (all-3.00 D nominally labeled power) as measured with an aberrometer off of the eye in a saline-filled wet cell. Lower order aberrations, including sphere and astigmatism have been zeroed to show only the higher order aberrations. Lenses are AirOptix for Astigmatism (AOA; Alcon, Ltd), Focus Dailies Toric (FDT; Alcon, Ltd., now discontinued), PureVision for astigmatism (PV; Bausch and Lomb, Inc.), and ACUVUE OASYS for astigmatism (AV; Johnson and Johnson Vision Care, Inc.). (Image courtesy Pete Kollbaum ${ }^{36}$ )

Of course, the combination of the eye plus contact lens optics are what provide the resulting vision for the wearer. Clinically, this resultant is typically verified by an in-office contact lens overrefraction, but there are several variables that may complicate this seemingly simple combination. As is well known, the eye contains inherent lower order (e.g. sphere and astigmatism) as well as higher order aberration. Several population studies have determined that many eyes have varying amounts of positive or negative higher-order aberration. However, due to some eyes having positive and other eyes negative amounts of these aberrations, when averaged across the population the resultant magnitude is largely zero. ${ }^{38}$ This is not true for spherical aberration, however, where the average ocular spherical aberration for the typical young adult population is positive and approximately 0.18 $\mu \mathrm{m}$ in magnitude (over a $6 \mathrm{~mm}$ pupil). ${ }^{39}$

Additionally, the lens itself could be made with aberration by chance or by design. Specifically, spherical contact lenses, by design, contain spherical aberration due to their highly curved anterior and posterior surfaces; minus lenses containing negative spherical aberration and plus lenses positive spherical aberration. ${ }^{40-42}$ Although less common today, manufacturing may also introduce some unintended aberration, such as by decentration of a mold, or as a consequence of the manufactured lens stabilization system. ${ }^{43}$

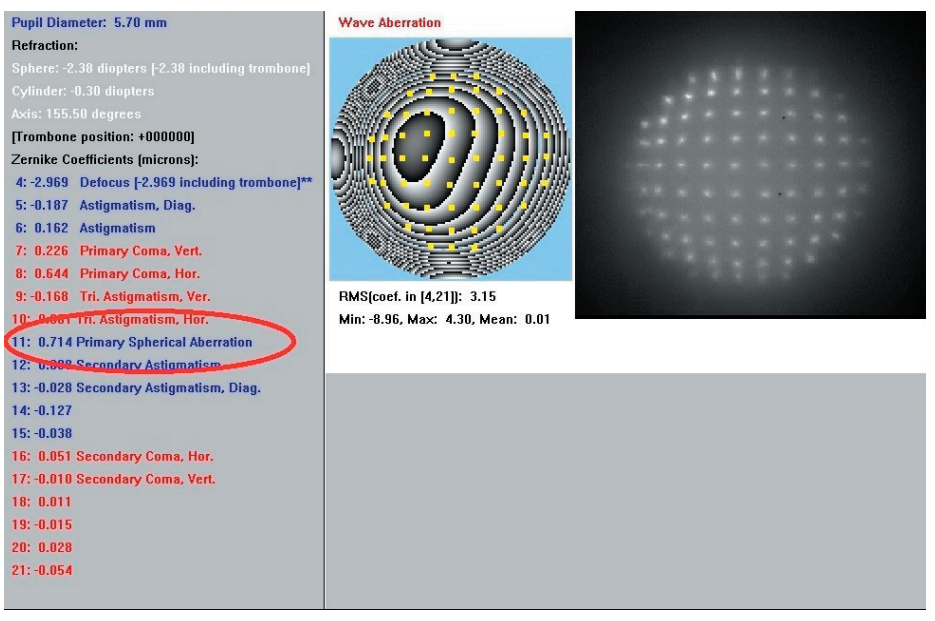

Figure 2. Early prototype Shack-Hartmann wavefront image and Zernike output for a positive spherical aberration multifocal aspheric soft contact lens on eye. Note that the highly variable spot size spacing leads to the high Zernike spherical aberration value. (Image courtesy lan (ox)

In theory, either the spherical aberration of the lens, the spherical aberration of the eye, or the spherical aberration of the eye plus lens could be corrected by creating a precise, radially symmetric asphericity in the contact lens. Interestingly, because of its inherent spherical aberration, a spherically surfaced soft contact lens of around $-7.00 \mathrm{D}$ has a magnitude of spherical aberration sufficient to counteract the average spherical aberration of the population.

Since substantial amounts of negative spherical aberration occur for powers as low as -4.00 D, many myopic contact lenswearing patients may have some spherical aberration corrected even by a typical single vision soft contact lenses. ${ }^{44}$ Those outside this range may experience little change, or a worsening, of their eye plus lens aberration. For instance, patients with positive defocus corrections (i.e. hyperopes) will experience even greater levels of positive spherical aberration, due to the combined effect of their inherent, ocular, spherical aberration and that induced by their spherical contact lens. Alternatively, it is possible to minimize the visual effects of spherical aberration by providing an appropriate, aspheric correcting surface on the contact lens, tailored for all defocus corrections. Indeed, several manufacturers (Bausch + Lomb, Cooper) did just this, starting in the late 2000's, by commercializing products, both spherical and toric, that used aspheric surfaces to minimize total eye plus lens spherical aberration for the average eye. The intention was to improve the quality of vision under low illumination conditions. 

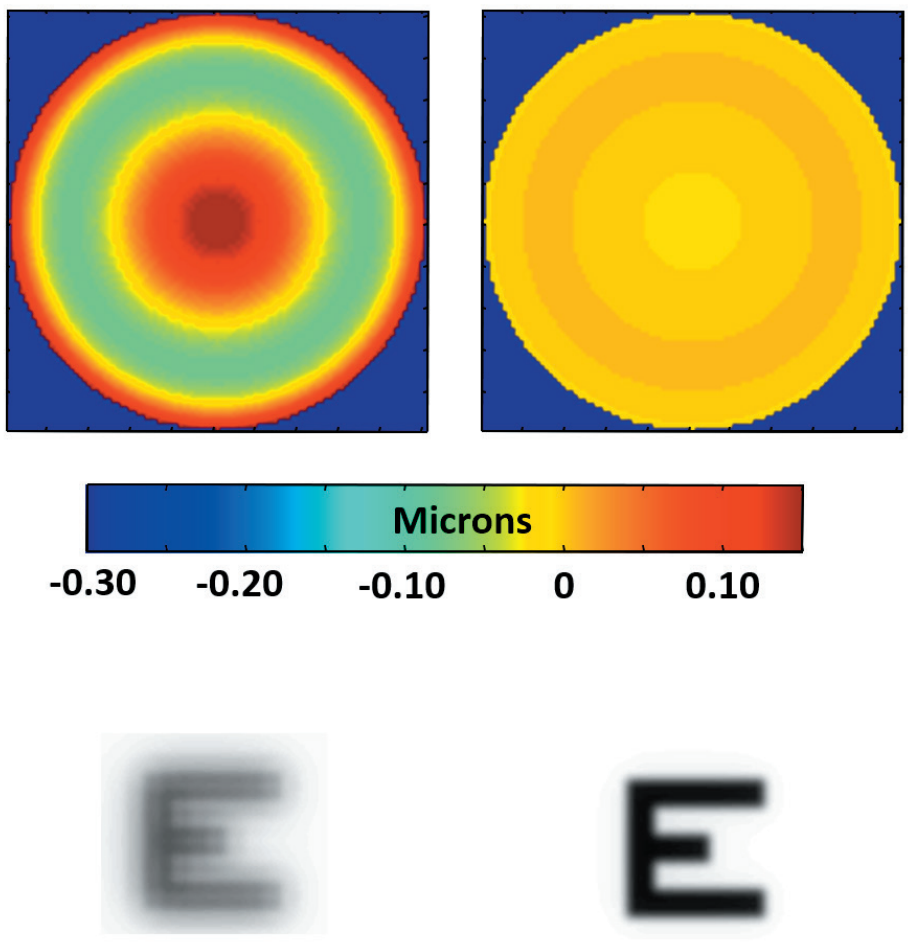

Figure 3. Higher order wavefront error (top row) and the resulting image simulations (bottom row) of the sum of the contact lens spherical aberration and that of the average eye while wearing a spherical lens that is of -1.00 D (left) and and-7.00 D power (right) (6 mm pupil). (Image courtesy Pete Kollbaum)

However, if this correcting lens (or any lens) is not centered on the eye, other visually degrading aberrations are introduced. Specifically, in the case of a lens with spherical aberration, coma is introduced in amounts directly proportional to the magnitude of the decentration. ${ }^{45}$ As decentrations can be as much as $1 \mathrm{~mm}$ horizontally and vertically in some lenses, ${ }^{46,47}$ this could have a substantial negative visual impact. Also, it is well-known that rigid lenses (e.g. corneal gas permeable) do not conform to the cornea, so introduce a tear film with optical power and aberration. However, although assumed to conform completely to the cornea, it has been shown that soft lenses do not always do so, but rather exhibit some partial flexure. This flexure, therefore, induces aberration when on the eye. For example, high power lenses may not induce the desired levels of sphere correction, ${ }^{48-50}$ and also induce unintended levels of spherical aberration. Specifically, they may introduce spherical power errors as high as 0.50 to 1.00 D, and spherical aberration errors up to around $0.20 \mu \mathrm{m}$ (for a $6 \mathrm{~mm}$ pupil). These levels of error are more than sufficient to degrade vision. Also, importantly, these levels of aberration are dependent on the lens power, thickness, and material/modulus. ${ }^{45}$

All the issues described above need to be addressed if the optical performance of the contact lens-wearing eye is to reach its maximum potential. Some manufacturers have attempted to minimize these issues. For example, aspheric lenses that have no spherical aberration as a function of lens power have been created to make them resistant to on-eye positioning errors and not introduce further aberration. Another approach combines the issue just described to create a lens that also accounts for on-eye flexure changes. ${ }^{51}$ Additionally, as mentioned above, while spherical aberration is clearly the largest higher-order ocular aberration in the general population, it is quite often not the dominant aberration in individual eyes. Third-order Zernike aberrations, such as coma and trefoil, are frequently problematic, even though their population average is close to zero. Unlike the approaches described above, these aberrations must be corrected by a rotationally stable contact lens, manufactured using a process capable of creating non-rotationally symmetrical surfaces. Clearly therefore, a contact lens must be designed to correct both symmetrical and non-rotationally symmetrical higher-order aberrations of the eye plus contact lens system, if visual benefit is to be maximized across a substantial proportion of the population.

Several research groups have attempted making customized higher-order wavefront aberration correcting soft contact lenses to minimize the effects of some of the problems described above. ${ }^{52,53}$ Due to the amounts of on-eye movement, even in soft contact lenses, limiting some of the achieved benefit, other groups continue working on similar concepts for scleral lenses with smaller on-eye movements. ${ }^{54,55}$ Results have been promising to date, with significant reductions in eye plus lens higher-order aberrations from these custom aberration-correcting contact lenses. Interestingly, however, the improvement in visual performance recorded by subjects wearing these lenses, while measurable, does not correlate well to the magnitude of higher order aberration reduction observed..$^{54}$ It has been demonstrated that some patients with chronic higher order aberrations, caused by corneal pathology, may adapt to their reduced retinal image quality and therefore, will require a period to "re-adapt" to the improvement provided by a custom correction. ${ }^{56}$

Clinical and laboratory wavefront sensors have also improved the design and manufacturing quality of multifocal contact lenses during the 2010's with most current designs incorporating multiple zones of continuous curvature to provide an increased depth of focus, from distance through intermediate and near.

These improved multifocal designs aim to better meet the visual needs of the current era in which computers and handheld electronic devices dominate the work environment, while simultaneously reducing the impact of glare and halos caused by sharp optical junctions between the power zones of previous lenses. In addition, many earlier presbyopic contact lenses were designed by adding the multifocal optical component to the single spherical surface of a single vision lens, without taking into consideration the inherent spherical aberration of the base lens design. This led to lenses having different optical power profiles depending upon their back-vertex power. ${ }^{54}$ Current generation multifocal lenses can be designed and tested with wavefront sensing metrology to ensure that all back-vertex powers have the same multifocal power profile across the optical zone. The resulting hope is that this leads to a consistent fitting experience for contact lens practitioners as they work with patients across a wide range of refractive corrections. Clinically, multifocal lenses now have an increased acceptance over monovision for presbyopic patients seeking a contact lens correction. This confirms the improved optical performance of these lenses that enhanced metrology and manufacturing has made possible.

A more recent application for lenses with designs partially derived from multifocal lens designs is the control of myopic 
progression. Individual clinicians have used bifocal and multifocal contact lenses as a treatment for myopia progression. They did so initially believing that the relaxation of accommodative demand at near would slow the development of myopia in the same manner that bifocal or progressive spectacles may be prescribed to children who developed myopia at an early age. More recent use relied on the theory of inducing peripheral retinal myopic defocus, to serve as a stop signal for the eye to continue to grow. ${ }^{57}$ Although large, controlled, longitudinal studies of myopic subjects wearing progressive spectacles showed little reduction in myopic progression, 58,59 soft concentric bifocal contact lenses with a central distance zone and peripheral near zone demonstrated a significant reduction in spherical equivalent refraction and axial length over time, compared to single vision contact lenses. ${ }^{60}$ These early designs were commercialized ${ }^{61}$ and cleared by the FDA in 2019 for use in the control of myopia progression. Alternative multifocal, aspheric and EDOF soft lens designs are also currently being tested clinically and are available commercially in some regions of the world for this same indication. The altered corneal shape induced by orthokeratology has also been shown to mimic the peripheral retinal defocus induced by these soft concentric multifocal designs, with a similar clinical impact on myopic progression, ${ }^{62,63}$ and is currently in use for myopia control in most parts of the world.

\section{CONCLUSION}

Contact lenses provide several unique optical advantages as a form of vision correction, particularly in terms of the increased field of view and freedom to enjoy many physical activities without the limitations that spectacles may create. Ironically, even though the primary reason for using contact lenses is refractive error correction, the early years of contact lens development were primarily devoted to balancing a comfortable, visually stable lens fit with a physiologically acceptable corneal environment. Development of toric and multifocal corrections occurred as manufacturing technology improved, but early on, in-vitro and in-vivo metrology was always the limiting factor for improving the optical performance of soft contact lenses. The more recent advent of wavefront sensors, capable of measuring the higher order aberrations of contact lenses in-vivo and in-vitro, has led to the exploration of improved visual performance of contact lens wearing eyes, for both normal and corneal distorted pathological eyes. Furthermore, these same developments in clinical and laboratory metrology have led to the design and manufacture of improved optical designs with enhanced goals of improving vision in some way, beyond that typically available. In particular, the enhanced design of multifocal contact lenses has led to a significant increase in the adoption of this modality by presbyopic patients, as well as somewhat similar designs to slow the progression of myopia:

\section{REFERENCES}

1. Pearson RM, Efron N. Hundredth anniversary of August Müller's inaugural dissertation on contact lenses. Surv Ophthalmol 1989;34:133-141.

2. Mandell RB. Contact Lens Practice, 4th ed. Springfield, IL: Charles C. Thomas, 1988.

3. Tuohy KM. Contact lens. US Patent 2,510,438, filed February 28, 1948.

4. Smelser GK, Ozanics V. Structural changes in corneas of guinea pigs after wearing contact lenses. AMA Arch Ophthalmol 1953; 49:335-340

5. Lambert SR, Klyce SD. The origins of Sattler's veil. Am J Ophthalmol. 1981 Jan;91(1):51-56.

6. Klyce SD. Stromal lactate accumulation can account for corneal oedema osmotically following epithelial hypoxia in the rabbit. J Physiol 1981;321:49-64.

7. Polse KA, Mandell RB. Critical oxygen tension at the corneal surface. Arch Ophthalmol 1970;84:505-508

8. Roscoe WR, Hill RM. Corneal oxygen demands: a comparison of the open- and closed-eye environments. Am J Optom Physiol Opt 1980;57:67-69.

9. Holden BA, Mertz GW. Critical oxygen levels to avoid corneal edema for daily and extended wear contact lenses. Invest Ophthalmol Vis Sci 1984;25:1161-1167.

10. O'Neal MR, Polse KA, Sarver MD. Corneal response to rigid and hydrogel lenses during eye closure. Invest Ophthalmol Vis Sci 1984;25:837-842.

11. Wichterle $\mathrm{O}, \mathrm{Lim}, \mathrm{D}$. Hydrophilic gels for biological use. Nature 1960;185:117-118.

12. Wichterle $\mathrm{O}$, Lim D. Process for producing shaped articles from three-dimensional hydrophilic high polymers. US Patent 2,976,576, March 28, 1961.

13. Lamb J, Bolton T. History of contact lenses. In: Phillips T, Speedwell L, eds. Contact Lenses, 6th ed. Elsevier 2019;Ch 1:12.

14. Newman S, Clamp J. Contact lens manufacturing. In Phillips T, Speedwell L, eds. Contact Lenses, 6th ed. Elsevier 2019; Ch 29:511.

15. Lamb J, Bolton T. History of contact lenses. In: Phillips T, Speedwell L, eds. Contact Lenses, 6th ed. Elsevier 2019;Ch 1:12.

16. Bennett ES, Weissman BA. Clinical Contact Lens Practice. Lippincott, Williams \& Wilkins, 2005; Ch.26:520.

17. Council BW Jr. Reciprocating tool holder assembly. US Patent 5,718,154, Feb 17, 1998.

18. Mandell RB. Contact Lens Practice, 4th ed. Springfield, IL: Charles C. Thomas, 1988.

19. Ellis D, Grimsrud R. Soft bifocal contact lenses for presbyopia (Thesis). Pacific University College of Optometry, 1983. https://commons.pacificu.edu/opt/637. 
20. Fleischmann W. The single vision reading contact lens. Am J Optom Arch Am Acad Optom 1968;45: 408-409.

21. Schor C, Landman L, Erickson P. Ocular dominance and the interocular suppression of blur in monovision. Am J Optom Physiol Opt 1987;64:723-730.

22. Loshin DS, Loshin MS, Comer G. Binocular summation with monovision contact lens correction for presbyopia. Int Cont Lens Clin 1982;9:161-165.

23. Erickson P. Potential range of clear vision in monovision. J Am Optom Assoc 1988;59:203-205.

24. Harris MG, Sheedy JE, Gan CM. Vision and task performance with monovision and diffractive bifocal contact lenses. Optom Vis Sci 1992;69:609-614.

25. Sheedy JE, Harris MG, Bronge MR, Joe SM, Mook MA. Task and visual performance with concentric bifocal contact lenses. Optom Vis Sci 1991;68:537-541.

26. Freeman MH. "Ophthalmic lens with diffractive power", US Patent 4,641,934 Feb 10, 1987.

27. Bradley A, Rahman HA, Soni PS, Zhang X. Effect of target distance and pupil size on letter contrast sensitivity with simultaneous vision bifocal contact lenses Optom Vis Sci 1993;70:476-481

28. Young G, Grey CP, Papas EB. Simultaneous vision bifocal contact lenses: a comparative assessment of the in vitro optical performance. Optom Vis Sci 1990;67:339-345.

29. Back A, Grant T, Hine N. Comparative Visual Performance of Three Contact Lens Corrections Optom Vis Sci 1992;69:474480.

30. Charman WN. Pinholes and presbyopia: solution or sideshow? Ophth Physiol Optics 2019;39:1-10.

31. Griffin RA. Multifocal ophthalmic lens with induced aperture. US Patent 6,474,814 B1 Nov 5, 2002.

32. Bakaraju RC, Ehrmann K, Ho A. Lenses, devices and methods of ocular refractive error. US Patent 9,201,250 B2 Dec 1, 2015.

33. Porter J, Guirao A, Cox IG, Williams DR. Monochromatic aberrations of the human eye in a large population. J Opt Soc Am A 2001;18:1793-1803.

34. Bakaraju RC, Ehrmann K, Ho A. Extended depth of focus contact lenses vs. two commercial multifocals: Part 1. Optical performance evaluation via computed through-focus retinal image quality metrics. J Optom 2018;11:10-20.

35. Lu F, Mao X, Qu J, Xu D, He JC. Monochromatic wavefront aberrations in the human eye with contact lenses. Optom Vis Sci 2003;80:135-141.

36. Kollbaum PS, Bradley A, Thibos LN. Comparing the optical properties of soft contact lenses on and off the eye. Optom Vis Sci 2013;90:924-936.

37. Kim E, Bakaraju RC, Ehrmann K. Reliability of power profiles measured on NIMO TR1504 (Lambda-X) and effects of lens decentration for single vision, bifocal and multifocal contact lenses. J Optom 2016;9:126-136.

38. Thibos LN, Hong Xin, Bradley A, Cheng Xu. Statistical variation of aberration structure and image quality in a normal population of healthy eyes. J Opt Soc Am A 2002;19:23292348.

39. Kingston AC, Cox IG. Population spherical aberration: associations with ametropia, age, corneal curvature, and image quality. Clin Ophthalmol 2013;7:933-938.

40. Westheimer G. Aberrations of contact lenses. Optom Vis Sci 1961;38:445-448.

41. Bauer GT. Longitudinal spherical aberration of modern ophthalmic lenses and its effect on visual acuity. Applied Optics 1980;19:2226-2234.

42. Cox IG. Theoretical calculation of the longitudinal spherical aberration of rigid and soft contact lenses. Optom Vis Sci 1990;67:277-282.

43. Kollbaum PS. Optical Aberrations of Contact Lenses and Eyes Corrected with Contact Lenses. PhD Thesis, Indiana University, 2007.

44. Dietze HH, Cox MJ. On- and off-eye spherical aberration of soft contact lenses and consequent changes of effective lens power. Optom Vis Sci 2003;80:126-134.

45. Guirao A, Williams DR, Cox IG. Effect of rotation and translation on the expected benefit of an ideal method to correct the eye's higher-order aberrations. J Opt Soc Am A 2001;18:10031015.

46. Tomlinson A, Ridder WH, Watanabe R. Blink-induced variations in visual performance with toric soft contact lenses. Optom Vis Sci 1994;71: 545-549.

47. Chen M, Sabesan R, Kamran Ahmad, Geunyoung Yoon, Correcting anterior corneal aberration and variability of lens movements in keratoconic eyes with back-surface customized soft contact lenses Opt. Lett 2007;32:3203-3205

48. Plainis S, Charman WN. On-eye power characteristics of soft contact lenses. Optom Vis Sci 1998;75:44-54.

49. Holden BA, Siddle JA, Robson G, Zantos SG. Soft lens performance models: the clinical significance of the lens flexure effect. Aust J Optom 1976;59:117-129.

50. Kollbaum PS, Bradley A, Thibos LN. Comparing the optical properties of soft contact lenses on and off the eye. Optom Vis Sci 2013;90:924-936.

51. Payor RE, Kollbaum P, Ye Ming, Bradley A. Optimizing optical aberrations in ophthalmic lenses. US Patent Application 2011/0102737 A1 May 5, 2011.

52. Marsack JD, Parker KE, Applegate RA. Performance of wavefront-guided soft lenses in three keratoconus subjects Optom Vis Sci 2008:85:1172-1178.

53. Sabesan R, Jeong TM, Carvalho L, Cox IG, Williams DR, Yoon G. Vision improvement by correcting higher-order aberrations with customized soft contact lenses in keratoconic eyes. Optics Letters 2007;32:1000-1002.

54. Marsack JD, Ravikumar A, Nguyen C, Ticak A, Doenig DE, Elswick JD, Applegate RA. Wavefront-guided scleral lens correction in keratoconus. Optom Vis Sci 2014;91:1221-1230.

55. Sabesan R, Johns L, Tomashevskaya O, Jacobs DS, Rosenthal 
P, Yoon G. Wavefront-guided scleral lens prosthetic device for keratoconus. Optom Vis Sci 2013;90:314-323.

56. Sabesan R, Yoon G. Perceptual learning after correcting the eye's aberration with adaptive optics. Invest Ophthalmol Vis Sci 2013;54: ARVO E-Abstract 1282.

57. Smith EL, Ramamirtham R, Qiao-Grider Y, Hung L-F, Huang J, Kee C, Coats D, Paysse E. Effects of foveal ablation on emmetropization and form-deprivation myopia. Invest Ophthalmol Vis Sci 2007;48:3914-3922.

58. Gwiazda J, Hyman L, Hussein M, Everett D, Norton TT, Kurtz D, Leske MC, Manny R, Marsh-Tootle W, Scheiman M. A randomized clinical trial of progressive addition lenses versus single vision lenses on the progression of myopia in children. Invest Ophthalmol Vis Sci 2003;44:1492-1500.

59. Berntsen DA, Sinnott LT, Mutti DO, Zadnik K. A randomized trial using progressive addition lenses to evaluate theories of myopia progression in children with a high lag of accommodation. Invest Ophthalmol Vis Sci 2012;53:640-649.
60. Anstice NS, Phillips JR. Effect of dual-focus soft contact lens wear on axial myopia progression in children. Ophthalmology 2011;118:1152-1161.

61. Ruiz-Pomeda A, Pérez-Sánchez B, Valls I, Prieto-Garrido FL, Gutiérrez-Ortega R, Villa-Collar C. MiSight Assessment Study Spain (MASS). A 2-year randomized clinical trial. Graefes Arch Clin Exp Ophthalmol 2018;256:1011-1021.

62. Cho P, Cheung SW, Edwards M. The longitudinal orthokeratology research in children (LORIC) in Hong Kong: a pilot study on refractive changes and myopic control. Curr Eye Res 2005;30:71-80.

63. VanderVeen DK, Kraker RT, Pineles SL, Hutchinson AK, Wilson LB, Galvin JA, Lambert SR. Use of orthokeratology for the prevention of myopic progression in children: A report by the American Academy of Ophthalmology. Ophthalmology 2019;126:623-636. 